\title{
Formación de profesionales en administración de negocios internacionales de cara a la Cuarta Revolución Industrial
}

\author{
Professional skills for International Business Graduates in the Fourth \\ Industrial Revolution
}

\author{
MSc. Rosberly Rojas Campos. \\ Universidad Estatal a Distancia, \\ Costa Rica \\ rrojas@uned.ac.cr
}

\author{
Mag. Helene Chan Castillo. \\ Universidad Estatal a Distancia, \\ Costa Rica. \\ hchang@uned.ac.cr
}

\author{
Mag. Karen Padilla Romero. \\ Universidad Estatal a Distancia, \\ Costa Rica \\ kpadilla@uned.ac.cr
}

\section{RESUMEN:}

El estudio analiza los requerimientos de las empresas en la formación de profesionales especializados en la gestión internacional; aspectos clave relacionados con conocimientos, formación técnica, habilidades y competencias para ejercer en el campo. La metodología contempla revisión documental, entrevistas y una encuesta al sector empresarial, por medio de un instrumento en línea. Se concluye que la formación de profesionales en negocios internacionales debe ser multidisciplinaria, contemplar el manejo de un idioma extranjero, fomentar el desarrollo de capacidades de razonamiento, liderazgo, comunicación, autonomía e innovación, e incorporar de manera transversal los contenidos de las tecnologías disruptivas para la generación de conocimientos y su aplicación por parte de los profesionales egresados.

\section{ABSTRACT:}

The study analyzes the requirements of companies regarding the training of professionals specialized in international management and identifies key aspects related to practical knowledge, technical training, skills and abilities. The methodology includes documentary reviews, interviews and a survey of the business sector via an online instrument. Therefore, the training of professionals in international business must be multidisciplinary and bilingual. Also, these need to develop their reasoning, leadership, communication, autonomy and innovation skills while taking advantage of disruptive technologies so that as graduates they may generate and implement knowledge.

\section{RESUMO:}

O estudo analisa os requisitos das empresas na formação de profissionais especializados em gestão internacional; aspectoschave relacionados ao conhecimento, treinamento técnico, habilidades e competências para exercer no campo. A metodologia inclui revisão documental, entrevistas e levantamento do setor empresarial, por meio de um instrumento on-line. Conclui-se que a formação de profissionais em negócios internacionais deve ser multidisciplinar, contemplar o conhecimento gestão de uma língua estrangeira, promover o desenvolvimento das capacidades de raciocínio, liderança, comunicação, autonomia e inovação e incorporar de forma transversal o conteúdo de tecnologias disruptivas para geração de conhecimento e sua aplicação por profissionais graduados

\section{RÉSUMÉ:}

L'étude analyse les demandes des entreprises en ce qui concerne la formation de professionnels spécialisés en administration internationale. Elle identifie des aspects clés liés aux connaissances, la formation technique, les habilités et les compétences pour exercer dans ce domaine. La méthodologie envisage la révision documentaire, les entrevues et un sondage du secteur de l'entreprise au moyen d'un instrument en ligne. Il ressort que la formation de professionnels en commerce international doit être multidisciplinaire et bilingue. De même, elle doit fomenter le développement de capacités de raisonnement, direction, communication, autonomie et innovation chez les professionnels tout en leur faisant incorporer de manière transversale l'utilisation de technologies de rupture, afin qu'ils puissent produire des connaissances et les mettre en pratique.

\begin{tabular}{l|l}
\multicolumn{1}{c|}{ PALABRAS CLAVES } & \multicolumn{1}{|c}{ KEYWORDS } \\
PERFIL PROFESIONAL, & PROFESSIONAL PROFILE, \\
HABILIDADES BLANDAS, & SOFT SKILLS, ABILITIES, \\
COMPETENCIAS, NECESIDADES & TRAINING NEEDS, INDUSTRY \\
DE FORMACIÓN, INDUSTRIA 4.0., & 4.O., INTERNET OF THINGS \\
INTERNET DE LAS COSAS (IOT). & (IOT).
\end{tabular}

\section{PALAVRAS CHAVES \\ PERFIL PROFISSIONAL, HABILIDADES PESSOAIS, HABILIDADES, NECESSIDADES DE TREINAMENTO, INDÚSTRIA 4.0., INTERNET DAS COISAS (IOT).}

\section{MOTS CLÉS}

PROFIL PROFESSIONNEL, HABILITES NON-TECHNIQUES, COMPETENCES, BESOINS DE FORMATION, INDUSTRIE 4.0., INTERNET DES OBJETS (IOT EN ANGLAIS). 


\section{INTRODUCCIÓN}

En la era del Internet de las Cosas (IoT), los cambios tecnológicos, las nuevas tendencias de consumo y la evolución de los roles que se desarrollan en la sociedad, resulta importante preguntarse cómo deben responder las universidades, desde su rol como academia, con una propuesta de formación que permita a los profesionales del futuro insertarse de manera exitosa en la sociedad 4.0.

Según el Foro Económico Mundial (World Economic Forum, 2016), la cuarta revolución industrial ha favorecido, junto con otros factores, un cambio en el modelo de negocio de las industrias a nivel global, generando nuevos paradigmas que inciden en sus exigencias en términos de las características y competencias que requerirán los puestos laborales en el futuro.

De cara a estos cambios, es necesario desarrollar nuevas habilidades y destrezas orientadas hacia la solución de problemas, inteligencia emocional, toma de decisiones, colaboración, negociación y flexibilidad cognitiva, etc. (Hecklau, Galeitzke, Flachs \& Kohl, 2016; Gronau, Ullrich \& Teichmann, 2017; World Economic Forum, 2016).

La Cuarta Revolución Industrial (Industria 4.0), en conjunto con el proceso expansivo de la globalización y con ella los cambios en el desarrollo económico y comercial, han dado como resultado una necesidad inminente de abordar los negocios de manera distinta (Kimm, 2018; Salinas \& Aksoy 2006; Schwab, 2015). El desarrollo de las tecnologías de la información y la automatización de procesos afecta todos los departamentos de la empresa (ManpowerGroup,2018; Singh, 2010).

En el ámbito regional, a inicios de la década de 1990, los países de Centroamérica tomaron la decisión de orientar su crecimiento hacia el comercio exterior (FLACSO, 2002; SIECA, CLACDS/INCAE, 1999). La tendencia hacia la formalización de acuerdos de libre comercio de orden regional y bilateral ha buscado la reducción de costos entre países miembros, dando como resultado acuerdos de amplio espectro que van más allá de simples concesiones de orden arancelario (UNCTAD, 2017). Adicionalmente, no hay que olvidar que, por su localización, Centroamérica, tiene una ventaja estratégica en el sistema de comercio internacional.

De manera complementaria, la Agenda 2030 (Organización de Naciones Unidas, 2015) recalca la importancia de robustecer el sistema de comercio y promover el intercambio comercial, sobre todo para los países en vías de desarrollo, con el propósito de fortalecer el crecimiento económico de manera sostenible, basado en normas de equidad y transparencia en la búsqueda del beneficio mundial. En este sentido, la Comisión Económica de Naciones Unidad para América Latina y el Caribe (CEPAL) hace referencia a un incremento en el dinamismo de la región, debido, entre otras cosas, a la demanda interna (inversión y consumo), donde el consumo privado fue favorecido por una menor inflación y un mayor ingreso disponible (CEPAL, 2016).

En relación con Costa Rica, la CEPAL (2016) reafirma que, a pesar de las crisis económicas y la marcada retracción de las actividades de intercambio comercial a nivel mundial, las políticas económicas claras y contundentes le han permitido lograr una recuperación importante e incluso se colocó a la cabeza en el crecimiento de este comercio exterior.

Ante este panorama mundial y regional, la Universidad Estatal a Distancia (UNED) de Costa Rica ha atendido las necesidades de formación en el área desde la carrera de Administración de Empresas con Énfasis en Negocios Internacionales, en el nivel de bachillerato desde 2006 y en el de licenciatura desde 2013 (Chan, Padilla \& Salas, 2011). La carrera se encuentra acreditada en el Sistema Nacional de Acreditación de la Educación Superior (SINAES, 2019).

La carrera se orienta principalmente hacia las áreas de la administración, el comercio internacional y un fuerte componente de investigación; estas son complementadas con la formación en ciencias económicas, mercadeo y aduanas. 


\section{METODOLOGÍA}

La metodología es de enfoque concurrente mixto, en el cual los datos cuantitativos y cualitativos se vinculan y analizan simultáneamente con el fin de responder a los objetivos del estudio (Hernández, Fernández \& Baptista, 2014; Pereira, 2011). A partir del análisis del entorno laboral de las personas profesionales en negocios internacionales y de las opiniones que tienen las personas empleadoras acerca de la formación deseada en el campo, se determina la orientación que deben tener los estudios universitarios. Para la recolección de información, se realizó el análisis documental, se aplicó un cuestionario a personas empresarias y una entrevista semiestructurada a personas expertas en ciencias económicas.

Por medio del análisis documental de diversas fuentes bibliográficas, se identificaron tendencias actuales del mercado laboral vinculadas con los requerimientos de formación de profesionales universitarios en la carrera estudiada.

Además, se elaboró una base de datos específica para puestos relacionados con la carrera en estudio a partir de la publicación elempleo.com (http://www.elempleo.com/cr/). Se utilizó esta herramienta web, que publica más de cinco mil anuncios, debido a su amplia cobertura, acceso libre (no requirió pago ni inscripción) y respaldo de una empresa consolidada en el país. Además, las opciones de búsqueda permiten detallar los requisitos del puesto, tanto en formación como en competencias, lo cual era clave para seleccionar aquellos con al menos grado de bachillerato universitario en carreras relacionadas con los negocios internacionales, el comercio internacional y la administración de empresas internacionales. Con estos criterios, se identificaron 79 anuncios, 16 de los cuales correspondían propiamente al perfil de una persona graduada en NNII. Las variables que se analizaron fueron el sector económico de la empresa, el nombre del puesto, él área de trabajo, el nivel jerárquico, la formación requerida, el grado académico, las competencias y el manejo de software.

Por otro lado, se recurrió al análisis del estudio presentado por la empresa Deloitte Global (2018), el cual mide la preparación de los negocios y el Gobierno de cara a la Cuarta Revolución Industrial. Dicho estudio valora los resultados de un instrumento aplicado a 1600 ejecutivos en 19 países.

El análisis documental se complementó con la aplicación de entrevistas a personas expertas en temas económicos: uno de la Cámara de Comercio de Costa Rica y otra de la Coalición Costarricense de Iniciativas de Desarrollo (CINDE). Esta última es una organización no gubernamental $(\mathrm{ONG})$ dedicada a la atracción de inversión extranjera directa a Costa Rica, cuyas empresas constituyen un mercado potencial de empleo importante para las personas graduadas en la carrera.

Con el objetivo de obtener información de primera mano de personas empresarias (gerentes y directivos de alto nivel de la compañía) con respecto a los conocimientos y habilidades que debería tener un profesional en el campo, se elaboró un cuestionario electrónico en la plataforma de encuestas denominada Limesurvey, el cual se aplicó entre julio y agosto del 2018. Este versó sobre la relevancia de las áreas de conocimiento en la formación del profesional en negocios internacionales, la valoración de cualidades y competencias profesionales, los paquetes informáticos (software) y habilidades tecnológicas requeridos, y las debilidades percibidas en la formación al contratar personal.

El envío de cuestionarios se realizó en forma masiva a partir de un listado de contactos de la carrera de NNII de la UNED, ya que, como es de esperar en relación con esta fuente de información, la respuesta es muy baja debido al alto costo de oportunidad del tiempo y a la débil vinculación histórica de las universidades con el sector empresarial costarricense. Se obtuvieron 42 respuestas.

Para el análisis de la información recopilada, se emplearon las siguientes categorías de análisis:

- Conocimientos requeridos, los cuales contemplan los saberes que se espera cuente una persona profesional de la carrera. 
- Necesidades de formación en tecnologías, que incluyen los conocimientos y habilidades en las principales tecnologías informáticas empleadas en el desempeño profesional de las personas graduadas en la carrera.

- Competencias profesionales, que consideran las capacidades con las que debe contar una persona profesional para poner en práctica los conocimientos y habilidades, y valores del perfil profesional de la carrera en el ámbito laboral.

\section{DESARROLLO}

\section{Antecedentes sobre el contexto externo de los negocios internacionales}

Desde ya hace más de tres décadas, Costa Rica optó por un estilo de desarrollo hacia afuera, basado en el modelo de promoción de exportaciones, que considera al mercado externo como el motor de la economía (Oviedo, Sánchez, Lindert \& López, 2015).

El comercio total (exportaciones más importaciones) del país representó un 67,6\% del PIB en el 2017 (Banco Central de Costa Rica, 2019); superior al indicador para América Latina, que se situó en 42,6 \%, y para el grupo de países de ingreso mediano alto (al que pertenece el país), en un 46,6 \%, de acuerdo con los datos del Banco Mundial (2019). Adicionalmente, tal como lo indica el Anuario Estadístico de la Promotora de Comercio Exterior de Costa Rica, el crecimiento de las exportaciones y las importaciones se ha venido diversificando significativamente; para ese año, los dispositivos médicos representaron un $25 \%$ del valor exportado (PROCOMER, 2018).

En este contexto de apertura comercial, las empresas que incursionan en los mercados internacionales deben adoptar modalidades de negocios diferentes a aquellas a las que están acostumbradas a escala nacional, lo cual supone ciertos conocimientos sobre la realidad de la economía global y la forma como se deben hacer los negocios internacionales.

Asimismo, la vinculación a los mercados internaciones conlleva el cumplimiento de estándares de calidad, el benchmarking y el aprendizaje constante en la organización, para el mejoramiento de la productividad y la competitividad.

El dinamismo del comercio y los negocios internacionales requiere formas de optimización de esas relaciones de intercambio, en lo cual tiene un papel relevante la formación de profesionales capaces de comprender los cambios del entorno y actuar de forma proactiva y propositiva con acciones innovadoras y de mejoramiento de los procesos productivos y comerciales (Baena \& Hurtado, 2017; Colomé, González, Pahissa, Roca \& Vilalta, 2015).

Se hace cada vez más relevante la formación de profesionales que potencien las ventajas competitivas del país y convertirlas en generadores del desarrollo regional. Por medio de la actualización constante de los planes de estudio, en términos de habilidades y capacidades para los negocios, se busca que las personas graduadas implementen innovaciones que generen valor.

Los estudios muestran la importancia de que la formación de profesionales en negocios internacionales considere los campos económicos, políticos y sociales desde la negociación como eje articulador, de manera que le permitan interactuar en contextos multiculturales, para aprovechar sus ventajas (Buitrago, 2015; Vanegas, Restrepo \& González, 2015; Wang \& Stewart, 2001). En particular, entre los temas que deben estar presentes en el currículo, destacan los siguientes: el análisis de riesgo, el comercio internacional, la resolución de conflictos y diplomacia, los contratos o tratados comerciales públicos o privados, y la estrategia y gestión.

En lo que se refiere a las competencias, el incremento constante en el acervo de conocimiento mundial es uno de los retos que enfrentan las universidades en el mundo con respecto a la formación de sus profesionales, dado el impacto que la revolución 4.0 genera de cara a los nuevos requerimientos laborales (González-Páramo, 2017).

El cambio en la tecnología es tan acelerado que quienes estudian una carrera en el presente trabajarán con tecnología 
que aún no existe y se enfrentarán a problemas que aún no conocen. Ante ello, las personas trabajadoras tienen que comprometerse con un aprendizaje a lo largo de la vida, si quieren mantener sus empleos y lograr carreras exitosas (Arranz, Blanco, \& Miguel, 2017; González-Páramo, 2017; Schwab, 2015; World Economic Forum, 2016; World Economic Forum, 2018).

Ello, aunado a la robotización que afectará los mercados de trabajo, implica la incorporación en el currículum de asignaturas que fomenten habilidades de orden tecnológico.

Colet, Balgiu \& Zaleschi (2017) reconocen que en la era de la Industria 4.0 los profesionales deberán contar con habilidades blandas específicas que requieren necesariamente una revaloración de los programas de desarrollo profesional y capacitación.

Para ello, es importante plantear estrategias educativas que promuevan juegos de roles y coaching, entre otros, que favorezcan el desarrollo de competencias orientadas hacia la flexibilidad, asertividad, independencia y disciplina, con el fin de que el profesional pueda responder de manera ágil y rápida a los cambios (Benesova \& Tupa, 2017; Colet, Balgiu \& Zaleschi, 2017; Pernías, 2017).

Galindo, Ruiz \& Ruiz (2017) agregan la importancia de que la inmersión en la era digital debe fomentarse y desarrollarse más allá del uso de herramientas simples, orientando la formación hacia el aprovechamiento de aquellas que permitan la generación de conocimientos y contenido.

Siendo así, la formación de profesionales debe contar necesariamente con conocimientos basados tecnologías disruptivas, análisis de Big Data, inteligencia artificial, tecnologías 3D y tecnología en la nube, entre otras (Betz, 2016; Fitsilis, Tsoutsa \& Gerogiannis, 2018; Hecklau, Galeitzke, Flachs \& Kohl, 2016; Lee, Kao \& Yang, 2014).

En el área de conocimiento de las ciencias económicas, se valoran aquellas relacionadas con liderazgo, compromiso ético y honestidad, compromiso con la calidad, capacidad de trabajo en equipo y capacidad de aprender y actualizarse (CINDE, 2018; García, 2009; Gutiérrez, Kikut, Rodríguez, Navarro, Picado y Azofeifa, 2016; Milhauser \& Rahschulte, 2010; Puga \& Martínez, 2008; van Heugten, Heijne-Penninga, Robbe, Jaarsma \& Wolfensberger, 2017).

En virtud de lo anterior, es de importancia para las universidades formar profesionales integrales, lo cual conlleve a la comprensión, análisis y sensibilidad hacia aspectos relacionados con la manera en que la cultura, el idioma, los sistemas políticos, la geografía y los factores socioeconómicos globales afectan la forma de hacer negocios.

\section{Requerimientos de formación para profesionales en el campo de la administración de empresas internacionales en Costa Rica}

A continuación, se sintetizan los resultados de los análisis de la base de datos de Elempleo.com, la encuesta a empresarios y las entrevistas a personas expertas.

De la encuesta se obtuvieron 42 respuestas de empresarios, gerentes y, en general, personal de la alta dirección de las empresas consultadas. Es preciso subrayar que la encuesta tenía la posibilidad de contestarse de manera anónima. Además, no todas las personas contestaron la totalidad de las preguntas. La mayoría de las empresas a la que pertenecían fueron del sector comercio y servicios (25 de las 31 que respondieron), cuatro de ellas son del sector agrícola y 2 del industrial.

La revisión del sitio Elempleo.com permitió analizar 16 empleos considerados por las autoras como aquellos aplicables a personas graduadas de las carreras de negocios internacionales. De ellos, seis correspondían a empresas del sector servicios, cuatro a manufactura y los demás uno en cada uno de comercio al por menor, consumo masivo, educación, industria alimentaria, salud y tecnología.

La mayoría de los puestos solicitados correspondían al área de compras de la empresa (6), seguida por las áreas de 
comercio (5), mercado internacional (4) y distribución (1). Estos puestos generalmente son para niveles profesionales (10 de los 16), aunque se solicitaban tres para coordinador(a) y tres para niveles de dirección superior (gerente, director, jefatura). Además, 7 de los puestos solicitan el nivel de bachillerato y 9 el de licenciatura. Entre las profesiones requeridas, sobresalen las de administración, comercio internacional e ingeniería industrial (ver tabla 1).

\begin{tabular}{l|c}
$\begin{array}{l}\text { Número de puestos ofertados para profesionales en administración } \\
\text { de negocios internacionales en Elempleo.com por carrera solicitada }\end{array}$ \\
$\qquad$\begin{tabular}{l|c} 
Carrera \\
Administración
\end{tabular} \\
\hline Comercio Internacional & 2 \\
\hline Ingeniería Industrial & 7 \\
\hline Aduanas & 3 \\
\hline Mercadeo & 2 \\
\hline Economía Agrícola & 1 \\
\hline Negocios Internacionales & 1 \\
\hline Relaciones Internacionales & 1 \\
\hline Otras & 2 \\
\hline
\end{tabular}

Tabla 1. Número de puestos ofertados para profesionales en administración de negocios internacionales en Elempleo.com por carrera solicitada Fuente: Elaboración propia con base en datos de Elempleo.com. 
En relación con las competencias, 6 de los puestos solicitaron competencias en negociación, la más frecuente, seguida por los temas de logística, estrategia, servicio al cliente y procedimientos de exportación e importación. Otras mencionadas son calidad, capacidad analítica, compras, control interno, evaluación, gerencia, liderazgo, manejo de personal, marketing digital, mercados internacionales, normativa, políticas y ventas (ver tabla 2).

\begin{tabular}{|c|c|}
\hline \multicolumn{2}{|c|}{$\begin{array}{l}\text { Número de puestos ofertados para profesionales en administración de ne- } \\
\text { gocios internacionales en Elempleo.com según la competencia solicitada }\end{array}$} \\
\hline Competencias solicitadas & Cantidad \\
\hline Negociación & 6 \\
\hline Logística & 3 \\
\hline Estrategia & 2 \\
\hline Servicio al cliente & 2 \\
\hline Procedimientos de importación y exportación & 2 \\
\hline Otras & 13 \\
\hline Total & 28 \\
\hline
\end{tabular}

Tabla 2. Número de puestos ofertados para profesionales en administración de negocios internacionales en Elempleo.com según la competencia solicitada Fuente: Elaboración propia con base en datos de Elempleo.com. 
En cuanto al manejo de software especializado, sobresale el manejo de sistemas de información gerenciales para la planificación de recursos empresariales (ERP por sus siglas en inglés y SAP Systeme Anwendungen und Produkte en alemán) en los procesos productivos y de distribución. También se hizo mención del Customer Relationship Management (CRM por sus siglas en inglés), que se utiliza para incrementar las ventas a la clientela actual y potencial, por medio del análisis de los datos históricos de la interacción entre cliente y empresa.

Entre las herramientas generales, se hizo referencia al manejo de Office y de Internet. El idioma inglés fue mencionado como requisito en 12 de los 16 puestos.

En términos de las áreas de conocimiento, 5 de las temáticas (idiomas, comercio exterior y logística internacional, mercadeo, negocios y negociación internacional y economía) obtuvieron 50 \% o más de respuestas positivas con respecto a la pertinencia de su formación para una persona especializada en la administración de NNII (Figura 1).

Emprendimiento, calidad y derecho fueron las temáticas con menor porcentaje de respuesta en relación con la pertinencia de su formación. Áreas de conocimiento en relaciones internacionales, contabilidad y finanzas, administración y aduanas se mantuvieron por debajo del $50 \%$ de las respuestas, pero en un porcentaje cercano a él.

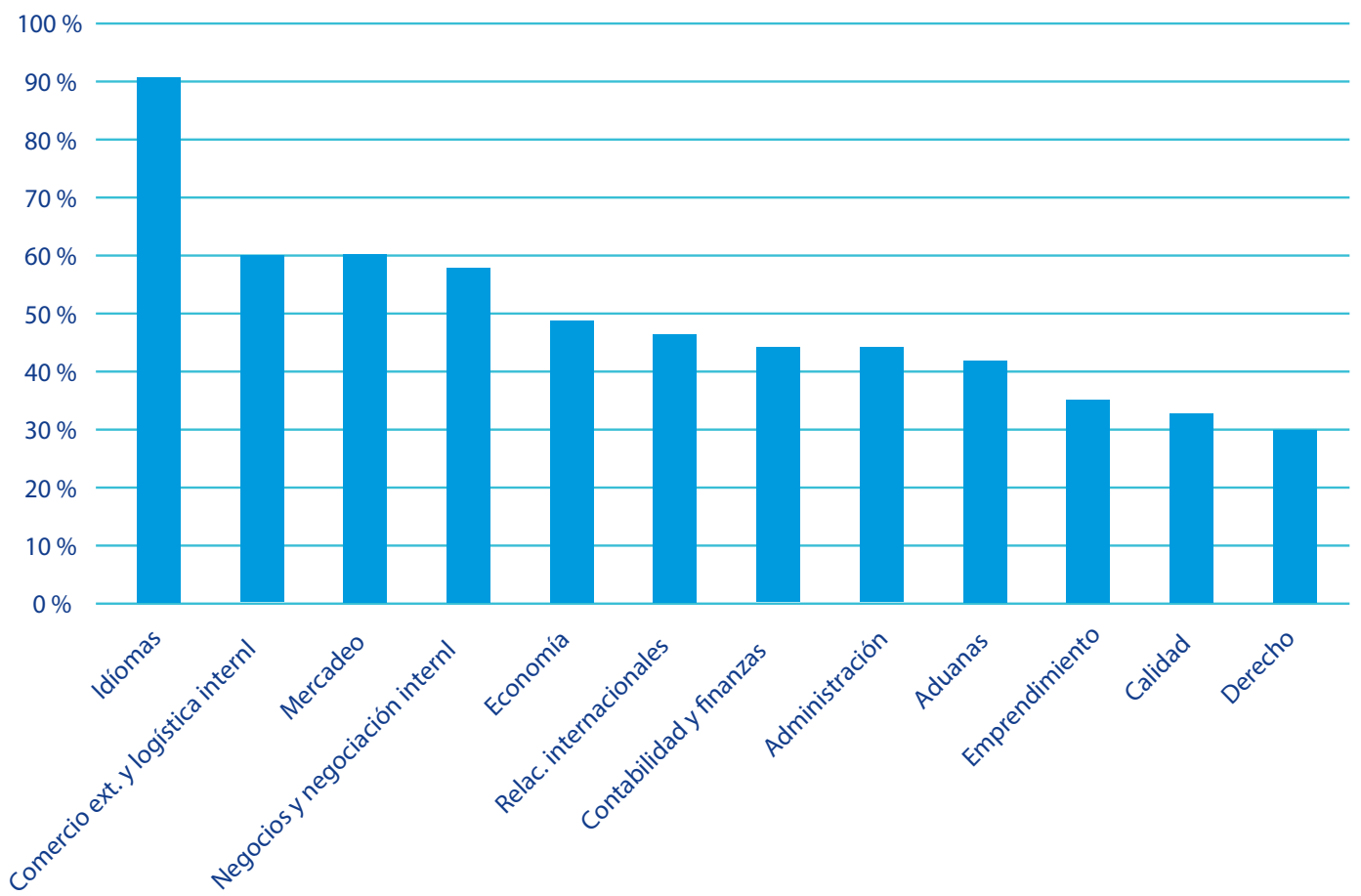

Figura 1. Valoración de la pertinencia de las áreas de conocimiento para la formación profesional en administración de negocios internacionales. (Porcentaje de respuestas por área)

Fuente: Elaboración propia con base en la encuesta a empresarios 
Como se observa en el Figura 1, los idiomas obtuvieron un 92,9\% de respuestas que los identifican como pertinentes. El idioma más frecuentemente mencionado fue el inglés. También se indicó la importancia del mandarín, el francés y el portugués. Sin embargo, no solo se mencionó un segundo idioma, sino también el manejo de un tercero.

En este sentido, hay coincidencia con lo encontrado por medio de las entrevistas a expertos, donde se resalta la necesidad de las empresas transnacionales de que sus profesionales de alto nivel cuenten con un manejo del idioma inglés a nivel de experto (C1 y C2 según el Marco Común Europeo de Referencia para las lenguas MCERL): “Administración de empresas es la carrera que gradúa más gente en el país, pero está en cuántas de estas personas hablan inglés, porque si no hablan inglés no pueden ingresar a estas empresas" (Salazar, D., comunicación personal, 20 de septiembre del 2018).

En la temática de comercio exterior y logística internacional (61,9 \% lo consideró relevante), se señaló la necesidad de contar con un buen conocimiento del sistema aduanero de la región y de la legislación aduanera internacional. Además, la experiencia en prácticas de importación y exportación, y el conocimiento de los requisitos y procesos, particularmente de países como Estados Unidos, China y los de Europa.

Los conocimientos en este campo deben permitir entender no solo los medios de transporte, sino cuál es la mejor opción en el proceso del comercio: "hoy las empresas lo que buscan es maximizar sus negocios y te contratan como profesional para que usted le resuelva dos cosas: ¿cómo soy más eficiente y cómo reduzco los costos?” (Chaves, J., comunicación personal, 27 de junio del 2018).

En relación con el comercio electrónico, como se señaló en las entrevistas, "este es fundamental en los bienes intangibles (los servicios), pero también como un canal de comercialización del bien; por tanto, hay que conocer toda su normativa y procesos, que son distintos al comercio tradicional (Chaves, J., comunicación personal, 27 de junio del 2018).

En el tema de mercadeo (61,9 \% lo valoró necesario) se recalcó la importancia del marketing digital y legislación respectiva en el uso de la web y redes sociales.

Los negocios y negociación internacional, como temática, se consideró relevante por el $59,5 \%$ de las personas entrevistadas. Se mencionó la capacidad y habilidad en la negociación, en particular precios y condiciones, lo cual está relacionado con las habilidades blandas; así como el manejo de las diferentes etapas de la negociación.

En concordancia, de las entrevistas se puede evidenciar "la necesidad de que la persona graduada en NNII reciba talleres de técnicas de negociación, ya que es importante no solo conocer sobre del producto que se comercia, sino también con quiénes se comercia, su cultura, costumbres, etiqueta de los negocios, entre otros temas" (Chaves, J., comunicación personal, 20 de setiembre del 2018).

El área de economía se consideró de importancia para el 50 \%; en específico, la capacidad de analizar el contexto económico para aplicarlo al negocio.

En el campo de las relaciones internacionales (es pertinente para el 47,6\%), se planteó la necesidad de contar con información básica sobre las culturas, forma de hacer negocios, diplomacia y etiqueta de los negocios.

El 45,2 \% de las personas que participaron en esta encuesta consideró relevantes los temas de contabilidad y finanzas, particularmente en lo relacionado con la creación de presupuestos, y el tema de la administración, en lo que atañe a la innovación en este campo.

En aduanas (para 42,9 \% fue relevante), las temáticas sugeridas fueron el manejo de trámites aduaneros (impuestos, desalmacenajes, costos, plazos de entrega, entre otros). En consonancia, las transacciones comerciales internacionales deben pasar por las aduanas; por tanto, se requiere conocer no solo el funcionamiento, sino también el derecho aduanero, entender el sistema multilateral de comercio y los organismos que juegan un rol importante: Organización Mundial de Comercio (OMC), la integración centroamericana y los organismos nacionales de comercio exterior (Chaves, J., comunicación personal, 20 de setiembre del 2018).

Un 35,7 \% juzgó como relevante el tema de emprendimiento. Se propuso realizar conversatorios con empresas con 
experiencia en negocios internacionales y el conocimiento sobre entidades gubernamentales que apoyan en este aspecto.

El 33,3 \% de las personas entrevistadas consideró como relevante la formación en metodologías Lean, Six Sigma y Green Belt, entre otras, así como procesos y control, vinculados con el tema de la calidad.

En el campo del derecho (31\% lo valoró pertinente), se señaló la importancia de temáticas del derecho nacional e internacional aplicado a la negociación. En general, se planteó la necesidad de talleres, prácticas y seminarios, que permitan acercar al estudiante con la realidad del campo profesional.

En el marco de tecnologías y programas informáticos, según las personas encuestadas, los programas básicos como el Office son fundamentales, sus 22 menciones superan por mucho los demás; pero, se señaló el manejo de Excel avanzado particularmente como una herramienta necesaria en los NNII. Otros programas importantes son los especializados, como los sistemas de información gerenciales para la planificación de recursos empresariales (ERP), entre los cuales se encuentra el SAP. En el caso del software TICA, para procesos de importación y exportación, se indicó la necesidad de acceso a prácticas en los cursos se la carrera.

Las redes sociales, en cuanto a su uso para impactar los negocios y el mercadeo, también se consideraron de importancia. Entre los programas de comunicación, se mencionaron aquellos de mensajería y conferencias, tales como Skype, Facetime, Whasapp, Telegram, etc.

En el cuestionario se consultó la importancia de un listado de competencias para una persona profesional de la carrera de Administración de Empresas con Énfasis en Negocios Internacionales. Para cada una de ellas, se solicitó la valoración con una escala de 1 a 5 , donde 1 correspondía a la importancia más baja y 5 a la más alta. La pregunta fue contestada por 32 personas.

La totalidad de las competencias consultadas recibió una alta valoración: todas mayores a 4. Como se observa en la tabla 8 , las que se consideraron de mayor importancia son las de compromiso ético y honestidad $(4,9)$, capacidad para identificar, plantear y resolver problemas $(4,8)$, manejo del inglés $(4,8)$, capacidad de aplicar los conocimientos en la práctica $(4,8)$, capacidad para trabajar en equipo $(4,8)$ y capacidad para tomar decisiones $(4,8)$.

Este resultado se complementa con lo mencionado en las entrevistas a personas expertas con respecto a la necesidad de que las personas graduadas demuestren tener habilidades blandas: "Las habilidades blandas deben incorporarse al currículo, se deben enseñar, evaluar; pero, sobre todo, que se pueda demostrar que el estudiante las ha adquirido para que esto le sirva en su vida laboral (Salazar, D., comunicación personal, 20 de setiembre del 2018). En esa misma entrevista se señalan como básicas las siguientes:

- Capacidad investigativa y de análisis lógico, crítico y estratégico.

- Toma de decisiones y resolución creativa de problemas.

- Atención al detalle: precisión, calidad, orden, excelencia en la presentación

- Liderazgo, comunicación efectiva, empoderamiento y compromiso, manejo de personal, capacidad de negociaciones complejas y especializadas.

- Comunicación en los negocios. Etiqueta de negocios, manejo del tiempo, comunicación oral y escrita, capacidad de síntesis, claridad, concreción ética, comunicación asertiva.

- Trabajo en equipo.

- Adaptabilidad. Tolerancia a la diversidad.

- Administración del tiempo y establecimiento de prioridades.

- Manejo del estrés. 


\begin{tabular}{l|c}
$\begin{array}{l}\text { Número de puestos ofertados para profesionales en administración de negocios interna } \\
\text { cionales en Elempleo.com según la competencia solicitada }\end{array}$ & Promedio \\
\hline \multicolumn{1}{|c}{ Competencia } & 4,9 \\
\hline Compromiso ético y honestidad & 4,8 \\
\hline Capacidad para identificar, plantear y resolver problemas & 4,8 \\
\hline Manejo del inglés & 4,8 \\
\hline Capacidad de aplicar los conocimientos en la práctica & 4,8 \\
\hline Capacidad para trabajar en equipo & 4,8 \\
\hline Capacidad para tomar decisiones & 4,7 \\
\hline Comunicación verbal & 4,7 \\
\hline Capacidad de adaptarse a situaciones nuevas & 4,7 \\
\hline Capacidad de aprender y actualizarse & 4,7 \\
\hline Capacidad para trabajar y organizar el tiempo & 4,6 \\
\hline Capacidad creativa e innovadora & 4,6 \\
\hline Compromiso con la calidad & 4,5 \\
\hline Capacidad para investigar en su disciplina & 4,5 \\
\hline Capacidad de análisis y síntesis & 4,5 \\
\hline Capacidad de analizar datos y convertirlos en información & 4,4 \\
\hline Manejo de programas informáticos básicos de oficina & 4,4 \\
\hline Capacidad para formular y gestionar proyectos & 4,4 \\
\hline Comunicación escrita & 4,3 \\
\hline Manejo de programas informáticos propios de su disciplina & 4,1 \\
\hline Capacidad de trabajar de forma autónoma & 4 \\
\hline & \\
\hline
\end{tabular}

Tabla 3. Promedio de la valoración de las personas consultadas sobre el grado de importancia de cada competencia en la formación profesional de la carrera de Administración de Empresas con Énfasis en Negocios Internacionales Fuente: Elaboración propia con base en la encuesta a empresarios.El listado de competencias incluido en la encuesta se basó en Gutiérrez, Kikut, Rodríguez, Navarro, Picado y Azofeifa (2016). 
Además, en relación con las competencias profesionales, una de las personas expertas indicó la necesidad de que la persona graduada en NNII reciba talleres de técnicas de negociación, ya que es importante no solo conocer sobre del producto que se comercia, sino también con quiénes se comercia, su cultura, costumbres y etiqueta de los negocios, entre otros temas (Chaves, J., comunicación personal, 27 de junio del 2018).

Finalmente, los empresarios se refirieron a carencias en competencias blandas (soft skills), como es el caso de debilidades en la toma de decisiones, la comunicación, el compromiso en el trabajo, la falta de habilidades para la innovación y la inteligencia emocional. Otro de los aspectos señalados por el empresariado fue la debilidad en el manejo de idiomas, particularmente del inglés y de las herramientas básicas de Office, en especial del Excel a nivel avanzado. Además de lo anterior, se resalta la falta de experiencia práctica en el campo de los NNII.

\section{CONCLUSIONES}

Las carreras en el campo de la administración de negocios internacionales tienen potencial de aumentar su matrícula por la pertinencia con el contexto nacional e internacional. En países como Costa Rica, con economías altamente vinculadas al comercio mundial, la formación en el campo puede contribuir al crecimiento económico inclusivo, la reducción de la pobreza y el desarrollo sostenible. Particularmente, los programas académicos deben estar permeados por principios éticos relacionados con un sistema de comercio internacional basado en normas, transparencia, inclusión y equidad.

Las ramas de los servicios y la manufactura son los sectores con mayor demanda de puestos en NNII, especialmente en las áreas de compras y de comercio.

En cuanto a la formación durante la carrera, los resultados del estudio muestran la necesidad de que sea multidisciplinaria. Se requiere la flexibilización de la malla curricular y el ofrecer especializaciones mediante asignaturas optativas. Todas las áreas del plan de estudios deben presentar casos reales, experiencia práctica, aplicaciones tecnológicas, talleres de negociación y conversatorios con personas empresarias del campo de negocios internacionales, entre otras.

El manejo avanzado de inglés debe ser parte del perfil de salida del profesional en NNII.

A partir de la síntesis de los datos recabados en las fuentes, se concluye que la formación en NNII debe fortalecer ciertas áreas. En el área del comercio internacional, se enfatiza en la logística y el comercio electrónico; esto último es congruente con la sugerencia de reforzar el área de mercadeo digital. Se sugiere fortalecer las áreas de aduanas y de legal en la formación de la carrera. Asimismo, se propone mayores capacidades de análisis económico. En lo relacionado con el área de administración, se sugiere una amplia variedad de temáticas, que abarcan desde las especializadas en negociaciones internacionales, hasta aspectos gerenciales necesarios para toda persona profesional en administración.

Se sugiere una nueva área relacionada con las relaciones internacionales, donde particularmente se estudien los temas de ética, cultura, diplomacia y resolución de conflictos internacionales.

Las habilidades blandas deben incorporarse al currículo de manera demostrable, es decir, que el estudiante las haya adquirido y las pueda aplicar a su vida laboral.

Del estudio se deriva una amplia gama de competencias y habilidades deseables para la persona profesional en NNII que abarcan capacidades de razonamiento, liderazgo, comunicación, relaciones interpersonales, ética, atención al detalle, autonomía, creatividad e innovación, resolución de problemas, trabajo en equipo, multitarea (multitasking) y sustentabilidad.

Existe coincidencia en cuanto a reforzar el manejo avanzado del Excel en la carrera de NNII, en vista de su uso en el 
campo laboral; en particular, se mencionan habilidades en fórmulas, tablas pivote, macros, gráficos y reportes.

Aunque, a estas alturas del siglo XXI, se podría suponer que cada persona graduada universitaria domina la suite ofimática Office para su desempeño estudiantil y posterior vida laboral, las fuentes de información de este estudio señalan la necesidad de que se cuente con esta herramienta, como una necesidad reiterada en el tema de TIC.

También, como coincidencia entre las fuentes de información, se plantearon necesidades de manejo de software especializado en los negocios, como los sistemas de información gerenciales para la planificación de recursos empresariales (ERP y SAP), principalmente.

Otra de las necesidades encontradas es el manejo de herramientas de Internet y redes sociales, especialmente aplicadas al desarrollo de los negocios y el mercadeo. Asimismo, programas de mensajería y comunicación como herramienta de desempeño laboral, como es el caso de Skype para reuniones

De manera complementaria, los estudios analizados describen una serie de elementos a considerar de cara la Revolución 4.0, entre ellos la necesidad de invertir en tecnología para orientar los nuevos modelos de negocio, fomentar la innovación más allá del desarrollo de productos y servicios, incorporando enfoques en áreas tales como talento, riesgo cibernético y disrupción competitiva; y, desde los programas de formación académica, fomentar una cultura de aprendizaje y colaboración orientada hacia las tecnologías de la información.

Las tecnologías emergentes han tomado un rol predominante en los nuevos modelos educativos, no solo como herramientas para facilitar los procesos de educación, sino también como aspectos relevantes que deben ser considerados en el planteamiento de los programas académicos de formación. El desarrollo del pensamiento crítico, el análisis de datos y la capacidad de hacer un uso eficaz de las tecnologías de la información hoy son requisitos para cualquier profesional, de cara a la Cuarta Revolución Industrial. 


\section{REFERENCIAS}

Arranz, F., Blanco, S. \& Miguel, F. (2017). Competencias digitales ante la irrupción de la Cuarta Revolución Industrial. Estudos em Comunicacao, 25, 1-11.

Baena, J. J. \& Hurtado, A. (2017). Generalidades de los negocios internacionales como programa de educación superior en Colombia. Science Of Human Action, 2(2), 269-291.

Banco Central de Costa Rica. (2019). Indicadores Económicos. Producto Interno Bruto, Gasto de Consumo Final, Formación Bruta de Capital, Exportaciones e Importaciones. Recuperado de https://gee.bccr.fi.cr/ indicadoreseconomicos $/$ Cuadros $/$ frmVerCatCuadro.aspx?idioma $=1 \&$ CodCuadro $=\% 202980$

Banco Mundial. (2019). Banco de datos. Indicadores del desarrollo mundial. Recuperado de https://databank. bancomundial.org/reports.aspx? source $=2 \&$ series $=$ NE.TRD.GNFS.ZS\&country $=$

Benesova, A. \& Tupa, J. (2017). Requirements for education and qualification of people in Industry 4.0. Procedia Manufacturing 11.

Betz, C. (2016). Renewing the IT Curriculum: Responding to Agile, DevOps, and Digital Transformation. Dymanic IT. Minessota State.

Buitrago, I. (2015). Tendencias de la disciplina en negocios internacionales. Revista Académica e Institucional Páginas de la UCP, 97, 109-121.

CEPAL (2016). Panorama de la Inserción Internacional de América Latina y el Caribe. Chile: ONU.

CINDE (2018). Demanda de Recurso Humano. Empresas de Inversión Extranjera Directa. San José, Costa Rica: CINDE.

Chan, H., Padilla, K. \& Salas, N. (2011). Plan de estudios de Administración de empresas con énfasis en Negocios Internacionales (Rediseño Bachillerato y Diseño Licenciatura). San José Costa Rica: Universidad Estatal a Distancia.

Colet, G., Balgiu B. \& Zaleschi, C. (2017). Assesment procedure for the soft skills requested by industry 4.0. MATEC Web of Conferences 121.

Colomé, R., González, S. Pahissa, E. Roca, M. \& Vilalta, M. (2015). Perfiles y competencias para la internacionalización. Barcelona, España: ESCI-UPF International Business. Recuperado de http://www.amec.es/wp-content/ uploads/2015/06/perfiles-y-competencias-para-la-internacionalizacion-amec-esci.pdf

Deloitte Global (2018). The Fourth Industrial Revolution is here. Are you ready? Deloitte Insights. Recuperado de https://www2.deloitte.com/insights/us/en/deloitte-review/issue-22/industry-4-0- technology-manufacturingrevolution.html

Fitsilis, P., Tsoutsa, P. \& Gerogiannis, V. (2018). Industry 4.0: Required Personnel Competences. International Scientific Journal Industry 4.0.

FLACSO (2002). Centroamérica en Cifras: 1980-2000. San José, Costa Rica: FLACSO.

Galindo, F., Ruiz, S. \& Ruiz, F. (2017). Competencias digitales ante la irrupción de la Cuarta Revolución Industrial. Revista Estudos em Comunicação 25(1), 1-11.

García, G. (2009). El concepto de competitividad sistémica. Revista Universidad de Sonora. México.

Gutiérrez, I., Kikut, L., Rodríguez, N., Navarro, G., Picado, C. \& Azofeifa, C. (2016). Empleadores 2013 de personas graduadas de universidades estatales. San José, Costa Rica: Consejo Nacional de Rectores. Oficina de Planificación de la Educación Superior. Observatorio Laboral de Profesiones. 
González-Páramo, J. (2017). Cuarta Revolución Industrial, empleo y estado de bienestar. Madrid: Real Academia de las Ciencias Morales y Políticas.

Gronau, N.; Ullrich, A. \& Teichmann, M. (2017). Development of the Industrial IoT Competences in the Areas of Organization, Process, and Interaction based on the Learning Factory Concept. Procedia Manufacturing 9, $254-261$.

Hecklau, F., Galeitzke, M., Flachs, S. \& Kohl, H. (2016). Holistic approach for human resource management in Industry 4.0. Procedia CIRP 54, 1-6.

Hernández, R., Fernández, C. \& Baptista, M. (2014). Metodología de la investigación. 6ta ed., México: McGraw-Hill. Kimm, S. (2018). Multilateral Trade Liberalization and Economic Growth, Journal of Economic Integration, 33(2), pp. 1261-1301

Lee, J., Kao, H. \& Yang, S. (2014). Service innovation and smart analytics for Industry 4.0 and big data environment. Procedia CIRP 6, 3-8.

ManpowerGroup. (2018). Robots Need Not Apply: Human Solutions for the Skills Revolution. New York, USA: Manpower Group. Recuperado de https://www.manpowergroup.com/wps/wcm/connect/59db87a7-16c6-490dae70-1bd7a322c240/Robots_Need_Not_Apply.pdf?MOD=AJPERES

Milhauser, K. \& Rahschulte, T. (2010). Meeting the needs of global companies through improved international business curriculum. Journal of Teaching in International Business 21, 78-100.

Organización de Naciones Unidas (2015). Transformar nuestro mundo: la Agenda 2030 para el Desarrollo Sostenible. Resolución aprobada por la Asamblea General el 25 de septiembre de 2015. New York: ONU. Recuperado de https:// unctad.org/meetings/es/SessionalDocuments/ares7od1_es.pdf

Oviedo, A., Sánchez, M., Lindert, K. \& López, H. (2015). Costa Rica’s development: from good to better. Systematic country Diagnostic. Washington, DC: Banco Mundial.

Pereira, Z. (2011). Los diseños de método mixto en la investigación en educación: Una experiencia concreta. Revista Electrónica Educare, XV(1), 15-29.

Pernías, P (2017). Nuevos Empleos, Nuevas Habilidades ¿Estamos preparando Talento para la cuarta revolución Industrial? ICE Revista de Economía 898, 59-71.

PROCOMER (2018). Estadísticas de Comercio Exterior Costa Rica, 2017. San José, Costa Rica: PROCOMER. Recuperado de https://procomer.com/downloads/estudios/estudio_estadistico_2017/Estadisticas2017.pdf

Puga, J. \& Martínez, L. (2008). Competencias Directivas en Escenarios Globales. Estudios Gerenciales, 24(109), 87103 .

Salinas, G. \& Aksoy, A. (2006). Growth before and after trade liberalization. World Bank Policy Research. Working Paper no. 4062, Washington, DC: The World Bank.

Schwab, K. (2015). The Fourth Industrial Revolution: What It Means and How to Respond. Recuperado de https:// www.weforum.org/agenda/2016/01/the-fourth-industrial-revolution-what-it-means-and-how-to-respond/

Singh, T. (2010). Does international trade cause economic growth? A Survey. The World Economy, 33(11), 15171564.

SIECA, CLACDS/INCAE (1999). El Corredor Logístico Centroamericano. Un Salto Cualitativo en la Dotación de Servicios al Comercio Exterior de la Región. El Salvador: SIECA, CLACDS/INCAE.

SINAES (2019). Carreras acreditadas. Recuperado de https://www.sinaes.ac.cr/index.php/home/carrera 
UNCTAD (2017). Evolución del sistema internacional de comercio y sus tendencias desde una perspectiva de desarrollo. Ginebra, Suiza: ONU.

Van Heugten, P., Heijne-Penninga, M., Robbe, P., Jaarsma, D. \& Wolfensberger, M. (2017). International perspectives on the profile of highly talented international business professionals. Journal of Education for Business, 92(7), 323331.

Vanegas, J. G., Restrepo, J. A. \& González, M. A. (2015). Negocios y comercio internacional: evidencias de investigación académica para Colombia. Revista Suma de Negocios, 6(13), 84-91.

Wang, J. \& Stewart, B. (2001). International Business Competencies Needed By Business Graduates in the United States and Taiwan, R.O.C. Paper presented at the Annual Meeting of the American Vocational Education Research Association. New Orleans, USA: AVERA.

World Economic Forum (2016). The Future of Jobs. Employment, Skills and Workforce Strategy for the Fourth Industrial Revolution. Ginebra, Suiza: WEF.

World Economic Forum (2018). Towards a Reskilling Revolution. A Future of Jobs for All. Ginebra, Suiza: WEF. 